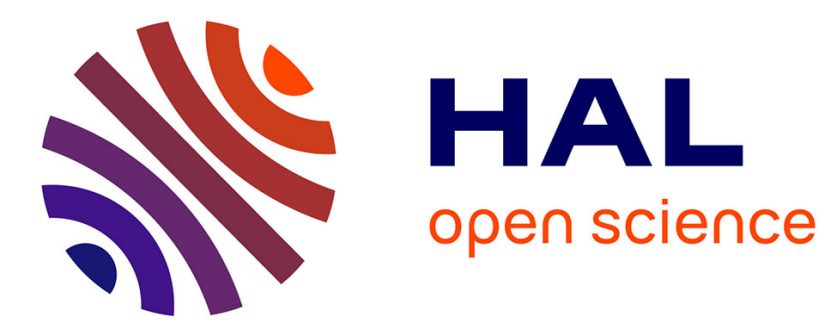

\title{
Maladie thromboembolique veineuse et thromboprophylaxie gravido-puerpérale
} Anne-Sophie Ducloy-Bouthors, Philippe Deruelle, Nathalie Trillot

\section{To cite this version:}

Anne-Sophie Ducloy-Bouthors, Philippe Deruelle, Nathalie Trillot. Maladie thromboembolique veineuse et thromboprophylaxie gravido-puerpérale. Praticien en Anesthésie Réanimation, 2019, 23, pp.139 - 144. 10.1016/j.pratan.2019.04.004 . hal-03486443

\section{HAL Id: hal-03486443 \\ https://hal.science/hal-03486443}

Submitted on 20 Dec 2021

HAL is a multi-disciplinary open access archive for the deposit and dissemination of scientific research documents, whether they are published or not. The documents may come from teaching and research institutions in France or abroad, or from public or private research centers.
L'archive ouverte pluridisciplinaire HAL, est destinée au dépôt et à la diffusion de documents scientifiques de niveau recherche, publiés ou non, émanant des établissements d'enseignement et de recherche français ou étrangers, des laboratoires publics ou privés.

\section{다)(1) $(5$}

Distributed under a Creative Commons Attribution - NonCommercial| 4.0 International 
Version of Record: https://www.sciencedirect.com/science/article/pii/S1279796019300567

Manuscript_69a3c5f128bf015df6ebafa54da379f7

Maladie thromboembolique veineuse et thromboprophylaxie gravido-puerpérale Thromboembolic venous disease and thromboprophylaxis during pregnancy

Anne-Sophie Ducloy-Bouthors ${ }^{1}$, Philippe Deruelle ${ }^{1}$, Nathalie Trillot ${ }^{2}$

Résumé : Les complications thromboemboliques sont la deuxième cause de décès maternel au cours de la grossesse. La stratégie de thromboprophylaxie qui a fait l'objet de recommandations doit être guidée par une appréciation du risque individuel. Le risque thrombotique peut être gradué en fonction des antécédents et des facteurs individuels clinique (âge) et biologiques. Les héparines de bas poids moléculaire constituent le traitement prophylactique de référence dont la posologie est adaptée au risque. La pratique d'une césarienne chez une parturiente sans facteur de risque ne justifie pas une thromboprophylaxie.

Summary : The second cause of maternal death during pregnancy is related to thromboembolic complications. The thromboembolic prophylaxis, which has been recently defined by guidelines, is graduated according to the thrombotic risk. The risk depends on patient clinical history, biological factors and individual factors (age). Low molecular weight heparin administration is the gold standard for thromboembolic prophylaxis during pregnancy. The heparin dose is determined according to the thrombotic risk. No thromboprophylaxis is required after caesarean section in parturient without risk factor.

Mots clés : grossesse, risque thrombo-embolique, thromboprophylaxie.

Key words : pregancy, thromboembolic risk, thromboprophylaxis

En France, la maladie thromboembolique veineuse (MTEV) est la deuxième cause de mortalité maternelle directe [1]. Entre 2010 et 2012, 24 décès maternels ont été attribués à des thrombo-embolies veineuses, soit un ratio de 1 pour 100.000 naissances (IC $95 \%$ 0,7-1,5). Les décès par complications thromboemboliques veineuses représentent 10,2 \% de l'ensemble des décès maternels et $22 \%$ de la mortalité maternelle directe dont cette complication la $2^{\text {ème }}$ cause. Les décès sont survenus dans $15 \%$ des cas (4/26) après une interruption de grossesse ( 1 interruption volontaire de grossesse et 3 interruptions médicales), $31 \%(8 / 26)$ pendant une grossesse évolutive (7 avant 22 SA et 1 à 39 SA) et $54 \%$ (14/26) après un accouchement [1].

${ }^{1}$ Maternité Jeanne de Flandre, Anesthésie Réanimation

${ }^{2}$ Service hémostase transfusion, CHU Lille 
L'incidence de la maladie thromboembolique veineuse pendant la grossesse et le postpartum est estimée à 1 pour 1000 dans les grandes enquêtes épidémiologiques scandinaves, se répartissant de façon égale entre les trimestres pour la thrombose veineuse profonde (TVP) et essentiellement en postpartum pour l'embolie pulmonaire (EP) [2].

Les deux dernières décennies de recherche clinique ont permis d'affiner l'appréciation du risque thromboembolique veineux et d'acquérir une meilleure connaissance des facteurs de risque de thrombose. Plusieurs auteurs ont développé des scores de risque efficaces pour guider la stratégie de prévention [3]-[5]. Des recommandations issues de consensus d'experts sur la thromboprophylaxie des patientes à risque sont à présent disponibles [5]-[9]. Ces recommandations ont eu un impact de réduction de la mortalité due à la thrombose au Royaume-Uni [10]. Pourtant, pour une même patiente, les préconisations recommandées sont très différentes et la conduite à tenir est parfois difficile à définir et à évaluer [11]. En effet, le niveau de preuve est souvent insuffisant pour apporter des recommandations fortes et de grandes études nécessiteraient un nombre de sujets et une uniformisation de la prise en charge au-delà du faisable [12].

L'objet de ce texte n'est pas de graduer la pertinence de chaque recommandation mais de partager une méthode, un guide qui permette de construire, pour la population de son propre bassin de vie, son propre protocole de prévention de la MTEV et d'évaluer son impact sur notre objectif primaire : la réduction de la morbidité et de la mortalité maternelle due à cette cause directe.

Cette méthode implique une détection et une évaluation du risque par l'ensemble des intervenants de la périnatalité dès le début de la grossesse ou même en pré conceptionnel. Pour la plupart des patientes pour lesquelles un risque est identifié, le protocole de prévention pourra être appliqué simplement. Mais pour certaines pathologies ou circonstances spécifiques, une réflexion pluridisciplinaire (obstétricien, anesthésiste, hématologue, médecin traitant, médecin vasculaire, interniste) sera nécessaire pour définir la conduite à tenir personnalisée.

La trame de ce texte liste les différentes recommandations à la disposition des lecteurs puis défile les questions cliniques pratiques qui ont permis l'élaboration du protocole de soins d'une maternité de recours maternel pour la population de son bassin de vie.

Liste des recommandations de pratique clinique pour la thromboprophylaxie au cours de la grossesse et du postpartum 
Les recommandations françaises sont issues d'une conférence de consensus multidisciplinaire de 2003 [13]. La SFAR a formalisé, en 2005, les recommandations pour la prévention de la MTEV qui incluait un chapitre sur la MTEV au cours de la grossesse et du postpartum [14]. Plus récemment, dans les dernières recommandations sur la prise en charge des patientes en postpartum, le CNGOF a émis un chapitre concernant la thromboprophylaxie [7].

Les recommandations internationales sont basées sur les données disponibles dans la littérature. Elles sont mises à jour régulièrement. Les guidelines du Royal College of Obstetrics and Gynaecology "Reducing the Risk of Venous Thromboembolism during Pregnancy and the Puerperium" ont été actualisées en 2015 [5]. Elles approfondissent de façon précise les odds ratio estimés pour les différents facteurs de risque et servent de référence pour de nombreuses autres recommandations nationales. Les dernières recommandations de l'American College of Chest Physicians : "VTE, Thrombophilia, Antithrombotic Therapy, and Pregnancy " datent de 2012 et guident de façon détaillée le traitement et la prévention de la MTEV pendant la grossesse et le postpartum [6]. Les recommandations de l'ESA de 2016 portent sur la chirurgie au cours de la grossesse et du postpartum [8]. Plus récemment, l'American Society of Hematology a émis des "Recommendations for VTE in the Context of Pregnancy" [9]. Son analyse insiste sur la difficulté à graduer les recommandations du fait du manque de données scientifiquement validées.

Cet écart de force et de contenu entre les différentes recommandations rend l'exercice complexe et parfois confus, même pour les spécialistes. Il est nécessaire de s'approprier l'appréciation du risque thrombotique et la conduite à tenir sous forme du protocole de soins pour la population prise en charge. Cette démarche est décrite dans deux articles récents dans lesquels la stratégie de thromboprophylaxie, guidée par les scores d'appréciation des risques a priori, a été évaluée [3][4]. Le score multidisciplinaire lyonnais a porté sur le suivi de 445 patientes dont 279 avait un antécédent personnel de thrombose. La thromboprophylaxie par HBPM a été prescrite chez $64,5 \%$ des patientes en prépartum et chez toutes les patientes 6 semaines en postpartum. Deux TVP $(0,73 \%)$ compliquent la période gravidique (une patiente ayant un score inférieur à 3 et une autre ayant un score supérieur à 6) et 4 TVP compliquent la période postpartum dont 3 chez des patientes ayant un score de 3 à 5 et une autre ayant un score supérieur à 6 . II n'y a pas eu d'embolie pulmonaire. Des saignements mineurs sont survenus dans $0,37 \%$ des cas [3]. La stratégie construite par Chauleur et coll. a également été évaluée récemment chez 2085 patientes à risque de thrombose et de pathologie vasculaire placentaire, avant et après l'implémentation du score de risque et de la stratégie associée [4]. En comparant les périodes avant et après, un évènement vasculaire est survenu chez 190 
$(19,2 \%)$ versus $140(13,0 \%)$ patientes (RR : 0.68 [0.55;0.83]). Le risque de TVP a été réduit (RR: $0.30[0.14 ; 0.67])$, de même que le risque de pathologie vasculaire placentaire: 79 versus 42 patientes (RR: $0.52[0.36 ; 0.75]$ ). Une hémorragie du postpartum est survenue chez 32 (3,2\%) versus 48 (4,5\%) patientes (RR $1 / 41.38$ [0.89; 2.13], $p$ 1/4 0.15) [4]. Ainsi, même si la synthèse des différentes recommandations semble complexe, l'élaboration et la mise en application d'un protocole de soins multidisciplinaire est un facteur de prévention du risque thromboembolique pendant la grossesse et le postpartum [3][4].

À titre d'exemple, le chapitre II décrit l'élaboration d'un tel protocole dans une maternité de recours maternel.

\section{Exemple d'élaboration d'un protocole de soins multidisciplinaire pour la population de recrutement d'une maternité de recours maternel}

Comment apprécier a priori le risque thrombotique veineux?

\section{Appréciation du risque}

Les scores de risque permettent à chacun des acteurs de la périnatalité d'identifier les facteurs de risque majeurs et mineurs et de les pondérer [2][3]. Cette appréciation du risque thrombotique cumulé est basée sur des odds-ratio d'excès de risque relatif, en comparaison avec la population sans facteur de risque [5]-[7]. Cette gradation du risque suppose donc que le risque de base de la population à bas risque du bassin de vie de l'établissement de soins ou du réseau périnatal, ait pu être évalué. Pour cette évaluation, des informations centralisées dans les réseaux thrombose sont recueillies: nombre d'évènements thromboemboliques veineux gravido-puerpéral sans facteur de risque identifié sur un territoire, et seront confrontées au nombre d'accouchements (naissances vivantes, interruption d'une grossesse de plus de 22 SA spontanée ou provoquée) ou de déclarations de grossesse si l'on s'intéresse au premier trimestre également.

La gradation du risque élaborée à partir de l'analyse des recommandations et des principaux points sur lesquels elles s'accordent :

- Certaines situations particulières induisent un risque très élevé et nécessitent des conduites à tenir personnalisées :

- Protocole multidisciplinaire personnalisé de soins pour chaque patiente présentant une maladie de système ou une pathologie médicale à haut risque de thrombose.

- Poursuite de l'anticoagulation pendant la grossesse et le 
postpartum pour les patientes sous anticoagulation au long cours.

- Ce sont avant tout les antécédents thrombotiques et leurs circonstances de survenue qui guident l'appréciation du risque [15] :

- Le risque thrombotique est classé comme risque élevé si l'antécédent thrombotique est récent, sans facteur déclenchant, récidivant ou s'il est survenu dans un contexte d'imprégnation hormonale: gravido-puerpérale ou sous œstro-progestatifs.

- Le risque thrombotique est classé comme risque intermédiaire si l'antécédent personnel de thrombose était non idiopathique et hors contexte de grossesse et d'œstro-progestatifs, ou distal.

- Le risque thrombotique est classé comme risque faible si l'antécédent personnel de thrombose est unique, avec facteur déclenchant, hors contexte gravido-puerpéral ou oestroprogestatif, ou qu'il existe un antécédent familial de premier degré.

Les facteurs de risque biologiques modulent cette gradation clinique. Ce sont les thrombophilies acquises et constitutionnelles (déficit quantitatif ou qualitatif en inhibiteurs physiologiques de la coagulation ou mutation des sites d'action de ces inhibiteurs). Le diagnostic de ces polymorphismes peut modifier l'évaluation du risque, en particulier si les mutations sont homozygotes ou composites [16].

Les facteurs de risque environnementaux liés ou non à la grossesse modulent l'appréciation du risque. Ce sont essentiellement l'âge, l'obésité, l'alitement prolongé, la procréation médicalement assistée, les antécédents de pathologie vasculaire placentaire, la multiparité et les grossesses multiples. Les recommandations du RCOG affectent des odds ratio à chacun de ces facteurs qui se multiplient au risque de base [5]. Dans le registre monocentrique de 10 années de suivi des patientes à risque de thrombose, ces facteurs additionnels ont toujours été retrouvés associés à l'évènement thrombotique lorsqu'il survient dans la population classée initialement comme à risque faible [17].

Les facteurs de risque en postpartum ont bien été identifiés dans les grandes études épidémiologiques scandinaves [2]: I'hémorragie du postpartum supérieure à $1000 \mathrm{~mL}$ ou ayant nécessité une transfusion multiplie le risque par 3 ; et la prééclampsie sévère et ses complications multiplie le risque par 5 . Le geste chirurgical de la césarienne en lui-même multiplie le risque par 3 , en particulier si elle est réalisée en urgence ou dans un contexte infectieux [2][8]. L'infection materno-foetale ou chirurgicale est elle aussi identifiée comme 
élevant le risque thrombotique en postpartum, en particulier le risque de thrombose veineuse ovarienne après césarienne. Dans le registre monocentrique, la moitié des embolies pulmonaires du postpartum concernaient des patientes prééclamptiques [17].

Deux circonstances particulières sont abordées dans les recommandations de I'ESA [8] : la chirurgie en cours de grossesse et les gestes chirurgicaux périnéaux.

Les actes chirurgicaux réalisés en cours de grossesse (kystectomie, gestes de chirurgie in utero) n'ont pas fait l'objet d'études concernant l'excès de risque thrombotique qu'ils génèrent. Ces gestes induisent de façon parfois prolongée le maintien en décubitus dorsal et un alitement. En l'absence de données scientifiques, les avis d'experts préconisent de guider la thromboprophylaxie sur le risque le plus élevé inhérent au terrain de la patiente ou à l'acte chirurgical pendant la période d'alitement.

Il n'y a pas de données dans la littérature pour guider les cliniciens concernant la thromboprophylaxie après un geste chirurgical péripartum (épisiotomie étendue, réfection de déchirure périnéale, drainage de thrombus). Au regard des recommandations appliquées dans la chirurgie urogénitale et pelvienne programmée, il paraît adéquat de prolonger la thromboprophylaxie tant que le syndrome inflammatoire n'est pas résorbé et la cicatrisation consolidée, ce qui peut prendre plusieurs semaines.

Les risques inhérents à la thromboprophylaxie antithrombotique pendant la grossesse et le postpartum

Le bénéfice de la prévention du risque de survenue ou de récidive de la MTEV doit être mis en balance avec les risques inhérents à la thromboprophylaxie: hémorragie, allergie, ostéoporose et thrombopénie induite à l'héparine. Dans la méta-analyse de Nelson-Piercy et Greer portant sur 15 études de patientes sous HBPM à dose curative et 61 études de patientes sous HBPM à dose préventive [18], la fréquence des complications hémorragiques est de $1,98 \%$ [IC95 1,5-2,6] dont hémorragies anténatales $0,43 \%$ [IC95 0,2-0,75] ; hémorragie du postpartum $0,94 \%$ [IC95 0,61-1,37]; hématome de paroi 0,61 \% [IC95 0,36-0,98]. La fréquence des complications allergiques est de 1,8\% [IC95 1,34-2,37], de l'ostéoporose de 0,04\% [IC 95 0,01-0,2] et celle de la thrombopénie de 0,11\% [IC95 0,02-0,32]. Cependant, celle de la complication majeure que constitue la thrombopénie induite à l'héparine apparait quasi-nulle 0,0 \% [IC95 0,0-0,11] [18]. Dans la méta-analyse de Romualdi et coll. portant sur 15 études, 3 abstracts, regroupant 981 femmes dont 822 traitées par héparine à dose curative, l'incidence des complications hémorragiques sévères $(\geq 1000 \mathrm{ml})$ pendant la grossesse est estimée à $1.41 \%(0.60-2.41 \%)$ et celle des complications 
hémorragiques sévères au cours des 24 premières heures du postpartum à 1,9\% (0.8-3.6\%) [19].

Comment prescrire une thromboprophylaxie gravidopuerpérale ?

\section{Quelle molécule ? Quelle dose ? Quand débuter et quelle durée ?}

Toutes les recommandations s'accordent sur le choix des héparines de bas poids moléculaire (HBPM) comme traitement de référence parce qu'elles associent efficacité et sécurité [5]-[8][13][14]. Plusieurs études de sécurité ont été réalisées sur l'emploi de plusieurs HBPM au cours des trois trimestres de la grossesse et du postpartum [18]-[22].

Trois grades de thromboprophylaxie peuvent être globalement identifiés :

- la dose curative : 175 à $200 \mathrm{UI} / \mathrm{kg}$ (en 1 ou 2 injections respectivement)

- pour les patientes à risquue très élevé

o pendant toute la grossesse et au minimum 6 semaines postpartum

- débutée en préconceptionnel ou dès le début de la grossesse par un relais de leur traitement anticoagulant au long cours ou la mise en route d'un traitement par HBPM

- la dose intermédiaire : $100 \mathrm{UI} / \mathrm{kg}$

- adaptée et prolongée

- pour les patientes à risque élevé et intermédiaire.

- dès le début et pendant toute la durée de la grossesse et au minimum 6 semaines postpartum

- ajustée au poids et au terrain

- La dose prophylactique : 4000 à $5000 \mathrm{UI}$

- pour les patientes sans facteur de risque qui sont exposées à un risquue transitoire

- pendant le temps d'exposition au risque.

Faut-il adapter la dose au poids et au terme de la grossesse?

Pour les doses curative et intermédiaire, les recommandations de RCOG proposent d'adapter la posologie au poids par classe [<50 kg ; 50-90 kg ; 91-130 $\mathrm{kg} ;>130 \mathrm{~kg}$ ] [5]. C'est le poids de début de grossesse qui est le plus souvent pris en considération. Cette adaptation doit être modulée par deux éléments : le gain de poids $>21 \mathrm{~kg}$ représente de façon indépendante un facteur de risque mineur [4] ; la pharmacocinétique des HBPM est modifiée par l'évolution de la grossesse et le syndrome inflammatoire [23]. 
II n'y a pas d'avantage pharmacocinétique à répartir la dose quotidienne en deux injections plutôt que une seule [23]. Cette répartition journalière a pu être préconisée pour les patientes obèses et avant l'accouchement pour faciliter la gestion péripartum de la thromboprophylaxie.

\section{Faut-il surveiller la numération plaquettaire, les D-Dimères et l'activité anti Xa?}

Selon les dernières préconisations ANSM, la numération plaquettaire doit être surveillée deux fois par semaine pendant un mois puis tous les mois chez les patientes à risque très élevé. Le dosage de l'activité anti Xa ou des D-Dimères, bien que non systématique, peut être proposé dans certaines situations cliniques, comme les pathologies systémiques associées, les poids extrêmes ou l'altération de la fonction rénale. La cible proposée en prévention doit être définie dans le protocole de soins : $0.2-0.6 \mathrm{IU} / \mathrm{ml}[6]$.

Faut-il prescrire une thromboprophylaxie après une césarienne programmée sans complication chez une patiente sans aucun facteur de risque ?

L'évolution de la médecine périopératoire autour de la césarienne a limité les procédures invasives (cathéter veineux et vésical) et leur durée, le saignement, les déséquilibres hydro-électrolytiques et la phase d'alitement. Cette réhabilitation précoce autorise à surseoir à la thromboprophylaxie postopératoire chez la patiente sans aucun facteur de risque après une césarienne programmée sans complication [7][8], même si la prudence reste de mise pour cette période postopératoire précoce d’hypercoagulabilité majeure [24].

\section{Gestion péripartum de la thromboprophylaxie}

Le maintien de la thromboprophylaxie augmente le risque d'hémorragie du postpartum et celui d'hématome intrarachidien compressif. Prévenir cette complication en cas d'anesthésie locorégionale requiert une fenêtre thérapeutique que le caractère imprévisible de l'accouchement rend difficile à gérer. La longueur de la fenêtre thérapeutique dépend de l'évaluation a priori du risque et de la dose administrée. Elle est préconisée par les experts à 12 heures en cas de dose prophylactique et 24 heures si dose intermédiaire ou curative [25][26].

\section{Conclusion}

La prévention du risque thrombotique veineux au cours de la grossesse et du postpartum repose sur le dépistage des patientes à risque. 
En l'absence d'études randomisées contrôlées, le niveau de preuve des recommandations reste faible mais elles correspondent à des accords professionnels d'experts qui se précisent d'année en année et justifient d'une appropriation par les équipes médicales en concertation multidisciplinaire.

Les modalités de prévention reposent sur les HBPM dont la date d'initiation et le schéma préventif, intermédiaire ou curatif, est à adapter au niveau de risque thrombotique et doit être régulièrement réévalué au cours de la grossesse, plus particulièrement dans le péripartum et dans le postpartum.

La stratégie individuelle retenue implique une information de la patiente sur les risques et bénéfices de l'attitude proposée, sur le choix des molécules, leurs modalités d'injection et sur l'aide à leur apporter compte tenu des contraintes.

\section{BIBLIOGRAPHIE}

[1] Rossignol, M., Morau, E., Dreyfus, M. Maternal death by venous thromboembolic disease. Anesth Reanim. 2018 ; 4 : 47-55.

[2] Jacobsen AF, Skjeldestad FE, Sandset PM. Incidence and risk patterns of venous thromboembolism in pregnancy and puerperium-a register-based case-control study. Am J Obstet Gynecol. 2008, 198 : 233-240.

[3] Dargaud Y, Rugeri L, Fleury C, Battie C, Gaucherand P, Huissoud C et al. Personalized thromboprophylaxis using a risk score for the management of pregnancies with high risk of thrombosis: a prospective clinical study. J Thromb Haemost. 2017, 15 (5) : 897-906.

[4] Chauleur C, Gris J-C, Seffert P, et al. Latest developments on risk factors and prophylaxis of thromboembolic disease in obstetrics. Gynecol Obstet Fertil. 2012, 40 : 301-7.

[5] Reducing the risk of venous thromboembolism during pregnancy and the puerperium. Green-top Guideline No. 37a April 2015. Royal College of Obstetricians and Gynaecologists. https://www.rcog.org.uk/globalassets/documents/guidelines/gtg37a.pdf.

[6] Bates SM, Greer IA, Middeldorp S et al. VTE, thrombophilia, antithrombotic therapy, and pregnancy: Antithrombotic Therapy and Prevention of Thrombosis, 9th ed: American College of Chest Physicians Evidence-Based Clinical Practice Guidelines. Chest. 2012, 141 (2 Suppl) : e691S-736s.

[7] Sénat MV, Sentilhes L, Battut A, Benhamou D, at al. Post-partum : Guidelines for clinical practice -Short text. J Gynecol Obstet Biol Reprod 
2015, 44, 1157-1166.

[8] Ducloy-Bouthors AS, Baldini A, Abdul-Kadir R, Nizard J. ESA VTE Guidelines Task Force. European guidelines on perioperative venous thromboembolism prophylaxis : Surgery during pregnancy and the immediate postpartum period. Eur J Anaesthesiol. 2018, 35 : 130-133.

[9] Bates SM, Rajasekhar A, Middeldorp $S$ et al. American Society of Hematology guidelines for management of venous thromboembolism : venous thromboembolism in the context of pregnancy. Blood Adv. 2018, 2 : 3317-3359.

[10] Cantwell R, Clutton-Brock T, Cooper G et al. Saving Mothers' Lives: Reviewing maternal deaths to make motherhood safer: 2006-2008. The Eighth Report of the Confidential Enquiries into Maternal Deaths in the United Kingdom. BJOG. 2011, 118, Suppl 1 : 1-203.

[11] Bates SM, Middeldorp S, Rodger M, James AH, Greer I. Guidance for the treatment and prevention of obstetric-associated venous thromboembolism. J. Thromb. Thrombolysis. 2016, 41 : 92-128.

[12] Rodger MA, Phillips $P$, Kahn SR, Bates $S$, McDonald $S$, Khurana R, James AH, Konkle BA, PROSPER Investigators: Tim Ramsay, Dean Fergusson, Anne McLeod, Wee Shian Chan, Rshmi Khurana, Kara Narenberg, Haim Abenhaim, John Heit, Ghada Bourjeilly, Paul Gibson, Kent Bailey. Low molecular weight heparin to prevent postpartum venous thromboembolism: A pilot study to assess the feasibility of a randomized, open-label trial. Thromb. Res. 2016, 142 : 17-20.

[13] Conférence de consensus. Thrombophilie et grossesse : prévention des risques thrombotiques maternels et placentaires. ANAES, 14 mars 2003, Paris. http://www.anaes.fr

[14] Benhamou D, Mignon A, Aya G et al. Prophylaxis of thromboembolic complications in obstetrics and gynaecology. Ann Fr Anesth Reanim. 2005, 24 : 911-20.

[15] Bezemer ID, Van der Meer FJM, Eikenboom JCJ et al. The value of family history as a risk indicator for venous thrombosis. Arch. Intern. Med. 2009, $169:$ 610-615.

[16] Bleker SM, Coppens M, Middeldorp S. Sex, thrombosis and inherited thrombophilia. Blood Rev. 2014, 28 : 123-133.

[17] Wibaut B, Trillot $N$, Deruelle $P$ et al. Preeclampsia as a risk factor for postpartum pulmonary embolism. Thrombosis Research. 2011, 127: S123-S150.

[18] Greer IA, Nelson-Piercy C. Low-molecular-weight heparins for thromboprophylaxis and treatment of venous thromboembolism in pregnancy: a systematic review of safety and efficacy. Blood. 2005, 106 : 401-407. 
[19] Romualdi E, Dentali F, Rancan E, et al. Anticoagulant therapy for venous thromboembolism during pregnancy: a systematic review and a metaanalysis of the literature. J Thromb Haemost. 2013, 11 : 270-81.

[20] Deruelle P, Denervaud M, Hachulla E et al. Use of low-molecular-weight heparin from the first trimester of pregnancy: a retrospective study of 111 consecutive pregnancies. Eur. J. Obstet. Gynecol. Reprod. Biol. 2006, $127: 73-78$.

[21] Nelson-Piercy C, Powrie R, Borg JY et al. Tinzaparin use in pregnancy: an international, retrospective study of the safety and efficacy profile. Eur. J. Obstet. Gynecol. Reprod. Biol. 2011, 159 : 293-299.

[22] Parent F, Deruelle P, Sanchez O, Meyer G, Girard P, Jilwan F, BoyerNeumann C, Wolf M, Simonneau, G. Safety of therapeutic doses of tinzaparin during pregnancy. Gynecol. Obstet. Invest. 2015, 79 : 256-262.

[23] Lebaudy C, Hulot JS, Amoura $Z$ et al. Changes in enoxaparin pharmacokinetics during pregnancy and implications for antithrombotic therapeutic strategy. Clin Pharmacol Ther. 2008, 84 : 370-377.

[24] Boyce H, Hume-Smith H, Ng J, Columb MO, Stocks GM. Use of thromboelastography to guide thromboprophylaxis after caesarean section. Int J Obstet Anesth 2011, 20 : 213-218.

[25] Horlocker TT, Wedel DJ, Rowlingson JC et al. Regional anesthesia in the patient receiving antithrombotic or thrombolytic therapy: American society of regional anesthesia and pain medicine evidence-based guidelines (3rd edn). Reg Anesth Pain Med. 2010, 35 : 64-101

[26] Gogarten W, Vandermeulen E, Van Aken $\mathrm{H}$ et al. Regional anaesthesia and antithrombotic agents:recommendations of the European society of anaesthesiology. Eur J Anaesthesiol 2010, 27 : 999-1015. 